\title{
Polymers of Substituted Acetylenes. II. Formation and Electrical Conductivity of Complexes of Poly(phenylacetylene)s*
}

\author{
Yoshinori Kuwane, Toshio MASUdA, and Toshinobu Higashimura \\ Department of Polymer Chemistry, Kyoto University, \\ Yoshida, Sakyo-ku, Kyoto 606, Japan.
}

(Received January 23, 1980)

\begin{abstract}
The formation of complexes of soluble poly(phenylacetylene)s [poly(PA)s] with various electron acceptors [2,3-dichloro-5,6-dicyano-p-benzoquinone (DDQ), 7,7,8,8-tetracyano-pquinodimethane (TCNQ), bromine, trifluoromethanesulfonic acid, and antimony pentachloride] was studied spectrophotometrically. The complexes showed an absorption in the region from 700 to $940 \mathrm{~nm}$. The ratios of the polymer to DDQ and to bromine in the complexes were determined to be $3: 1$. The $1: 1$ mixtures of poly(PA) with DDQ and with TCNQ showed conductivities of $c a .10^{-8}$ $\mathrm{ohm}^{-1} \mathrm{~cm}^{-1}$, whereas that of poly(PA) was as low as $10^{-14}-10^{-17} \mathrm{ohm}^{-1} \mathrm{~cm}^{-1}$. The $n$-type conduction was confirmed by thermoelectric-power measurement.
\end{abstract}

KEY WORDS Poly(phenylacetylene) / Polymer Complex / Electron Acceptor / 2,3-Dichloro-5,6-dicyano-p-benzoquinone / 7,7,8,8-Tetracyanop-quinodimethane / Absorption Spectroscopy / Electrical Conductivity / Semiconductivity /

There have been many studies on the preparation and electrical conductivity of charge-transfer complexes consisting of electron-donating polymers and low-molecular-weight electron acceptors. ${ }^{1,2} \operatorname{Poly}(\alpha-$ vinylnaphthalene $),{ }^{3}$ poly(vinylpyridine $) \mathrm{s},{ }^{4} \operatorname{poly}(N$ vinylcarbazole $)^{5}$ etc. have been used as electrondonating polymers; 2,3-dichloro-5,6-dicyano- $p$ benzoquinone (DDQ), 7,7,8,8-tetracyano-p-quinodimethane (TCNQ) etc. as organic electron acceptors; and halogens and Lewis acids as inorganic electron acceptors. These complexes are easily prepared by mixing the solutions of two components, and some of the resulting mixtures show semiconductivity.

It will be of interest if acetylenic polymers work as electron-donating polymers to form complexes with low-molecular-weight electron acceptors. In fact, when films of cis- or trans-polyacetylene, $(\mathrm{CH})_{n}$, are doped with electron acceptors such as halogens and arsenic pentafluoride, they show metallic con-

* Part I: T. Masuda, T. Ohtori, and T. Higashimura, Polym. J., 11, 849 (1979). ductivity up to $\sim 10^{2} \mathrm{ohm}^{-1} \mathrm{~cm}^{-1} .^{6-9}$ This suggests that polymers of various acetylene derivatives are able to form charge-transfer complexes with electron acceptors, and further that there is the possibility of the complexes formed being highly conducting.

Since polyacetylene is insoluble in any solvent, it is impossible to prepare its complexes in solution, and therefore a study on their stoichiometry is difficult. We have succeeded in synthesizing soluble polymers from phenylacetylene $(\mathrm{PA})^{10}$ and its derivatives. ${ }^{11}$ The poly(phenylacetylene) [poly(PA)] formed has a high molecular weight up to 15000 . Though Ziegler-type catalysts are also effective in bringing about the polymerization of PA, the main products are insoluble polymers. ${ }^{12,13}$

In the present study, the complexes obtained in solution from soluble poly(PA) and various electron acceptors (DDQ, TCNQ, bromine, trifluoromethanesulfonic acid, and antimony pentachloride) were investigated by means of spectrophotometry. Furthermore, the relationship between the electrical conductivity and composition of mixtures of poly(PA) with acceptors was studied. 


\section{EXPERIMENTAL}

\section{Materials}

Poly(PA) was synthesized by the polymerization of phenylacetylene catalyzed by $\mathrm{WCl}_{6}$ and $\mathrm{MoCl}_{5} \cdot{ }^{10}$ Methanol in an amount half as much as the catalysts was used as a cocatalyst. The polymerization was carried out at $30^{\circ} \mathrm{C}$ in benzene at $[\mathrm{M}]_{0}=1.0 \mathrm{~mol} \mathrm{dm}^{-3},[\mathrm{Cat}]=10-20 \mathrm{mmol} \mathrm{dm}^{-3}$ under a nitrogen atmosphere. $\mathrm{WCl}_{6} \cdot(1 / 2) \mathrm{CH}_{3} \mathrm{OH}$ afforded a trans-rich polymer [poly(PA)-W], whereas $\mathrm{MoCl}_{5} \cdot(1 / 2) \mathrm{CH}_{3} \mathrm{OH}$ gave a cis-rich polymer [poly(PA)-Mo]. ${ }^{14}$ In a similar manner, $\operatorname{poly}(\beta$ naphthylacetylene) $[\operatorname{poly}(\beta-\mathrm{NA})]^{15}$ and poly $(1-$ phenylpropyne) $[\text { poly }(\mathrm{PP})]^{16}$ were prepared by polymerization catalyzed by $\mathrm{WCl}_{6} \cdot(1 / 2) \mathrm{CH}_{3} \mathrm{OH}$ and $\mathrm{WCl}_{6} \cdot\left(\mathrm{C}_{6} \mathrm{H}_{5}\right)_{4} \mathrm{Sn}$, respectively.

DDQ was purified by sublimation; $\mathrm{mp} 217^{\circ} \mathrm{C}$ (lit. $\left.217-219^{\circ} \mathrm{C}^{17}\right)$. TCNQ was recrystallized from an acetonitrile solution, and then sublimed; $\mathrm{mp}$ 293.5-295 ${ }^{\circ} \mathrm{C}$ (lit. $293.5-296^{\circ} \mathrm{C}^{18}$ ). Chloranil was purified by column chromatography on calcium carbonate, recrystallized from a benzene solution, and then sublimed; $\mathrm{mp} 299^{\circ} \mathrm{C}$ (lit. $289.0-290^{\circ} \mathrm{C}^{19}$ ). Bromine, trifluoromethanesulfonic acid, and antimony pentachloride were used without further purification. The solvents for polymerization and for preparation of polymer complexes were purified as described elsewhere. ${ }^{10,14,15}$ Spectroscopic-grade ethylene dichloride was purified by distillation over calcium hydride in order to be used for measuring the absorption spectra.

\section{Absorption Spectroscopy}

Solutions of polymer complexes in ethylene dichloride were prepared under a nitrogen atmosphere; the total molar concentration of any polymer (based on monomer unit) and electron acceptor was $2.00 \mathrm{mmol} \mathrm{dm}^{-3}$. Absorption spectra were measured with a Hitachi Model EPS-3T spectrophotometer.

\section{Electrical Conductivity}

Solutions of a polymer and an electron acceptor in methylene dichloride were mixed together and agitated for $1 \mathrm{~h}$ at room temperature. The solvent was evaporated and the residue was dried under reduced pressure. The polymer complexes obtained were finely ground and pressed at $100 \mathrm{~kg} \mathrm{~cm}^{-2}$ for $30 \mathrm{~min}$ into pellets $(150-200 \mathrm{mg} ; 12 \mathrm{~mm}$ in diameter and $0.6-0.8 \mathrm{~mm}$ in thickness). Gold was evaporated with a JEOL Model JEE4X vacuum evaporator onto both sides of the pellets to make two terminal electrodes and one guard electrode. Copper wires were attached to the electrodes by silver paste (Fujikura Kasei Co., Dotite D-550). The electrical conductivity of the polymer complexes was determined by measuring the current at 20 $25^{\circ} \mathrm{C}$ with a Takeda-Riken Model TR-8651 electrometer. The thermoelectric-power measurement was carried out by just observing the direction of electric power, in comparison with that of an $n$-type germanium semiconductor.

\section{RESULTS AND DISCUSSION}

\section{Absorption Spectroscopy}

Figure 1 shows the absorption spectra of equimolar mixtures of poly(PA)-W with several electron acceptors measured in ethylene dichloride at a concentration of $1.0 \mathrm{mmol} \mathrm{dm}^{-3}$ for each component.

The solution of poly(PA)-W in ethylene dichloride $\left(1.0 \mathrm{mmol} \mathrm{dm}^{-3}\right)$ was red, and exhibited absorptions ranging from the ultraviolet region to $c a$. $670 \mathrm{~nm}$. The solution of DDQ in ethylene dichloride $\left(1.0 \mathrm{mmol} \mathrm{dm}^{-3}\right)$ was yellow and showed no absorption where the wavelength was greater than $600 \mathrm{~nm}$. On the other hand, a solution of a mixture of poly(PA)-W and DDQ was dark yellow, and gave rise to a new absorption at $940 \mathrm{~nm}$ (see

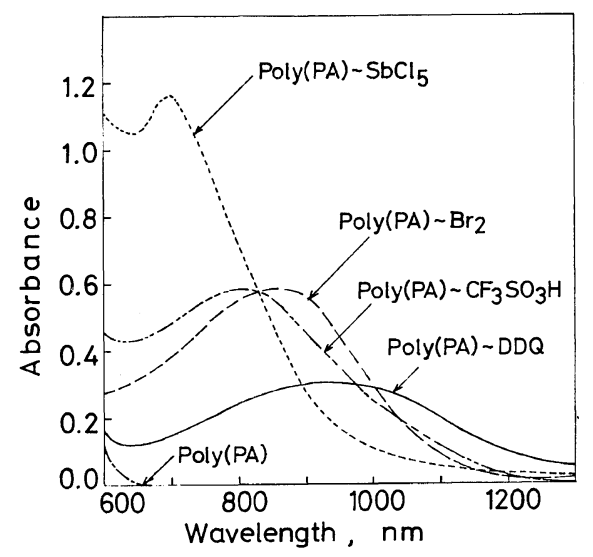

Figure 1. Absorption specta of $1: 1$ mixtures of poly(PA)-W with several electron acceptors in ethylene dichloride solutions; total concn, $2.0 \mathrm{mmol} \mathrm{dm}^{-3}$. 
Figure 1). A similar but smaller absorption was observed when TCNQ was used as another electron acceptor.

Similar absorptions were observed as well, when bromine, trifluromethanesulfonic acid, and antimony pentachloride were used as electron acceptors. The maxima of absorptions were situated at 860 , 800 , and $700 \mathrm{~nm}$, respectively. None of the acceptors had any absorption where wavelength was greater than $600 \mathrm{~nm}$.

Poly(PA)-Mo also gave similar absorptions due to the formation of complexes with acceptors, but their absorbances were smaller.

The composition of complexes was evaluated by the continuous variation method (Figure 2). Both mixtures of poly(PA)-W with DDQ and with bromine showed the largest absorption where the acceptor content was $25 \mathrm{~mol} \%$. This means that every third unit of poly(PA)-W interacts with one molecule of acceptor to yield the complexes.

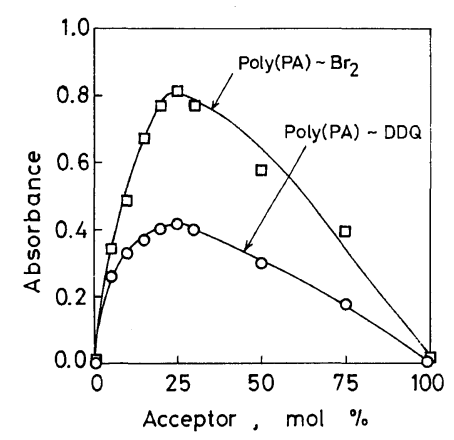

Figure 2. Estimation of composition of complexes by the continuous variation method; in $\left(\mathrm{CH}_{2} \mathrm{Cl}\right)_{2}$, total concn, $2.0 \mathrm{mmol} \mathrm{dm}^{-3} ; \lambda_{\max } 940 \mathrm{~nm}$ for poly(PA)-W DDQ, $860 \mathrm{~nm}$ for poly(PA)--W $\sim \mathrm{Br}_{2}$.

When poly(PA)-W and an excess of bromine were mixed in a carbon tetrachloride solution, and the solvent and free bromine were evaporated, a complex with the polymer-to-bromine ratio of $c a$. 4:1 was isolated, as is also indicated in the literature. ${ }^{20}$ This lower content of bromine in the isolated complex seems due to the loss of less tightly bound bromine.

The above results reveal that the soluble poly(PA) obtained with $\mathrm{WCl}_{6}$ or $\mathrm{MoCl}_{5}$ produces complexes with various acceptors, although poly(PA) contains neither an aromatic fused ring nor an element with lone-pair electrons such as nitrogen. Formation of a complex even from a Brönsted-acid like trifluoromethanesulfonic acid suggests that the complexes formed might be appreciably polarized or virtually ionized.

\section{Electrical Conductivity}

Figure 3 shows the dependence of electrical conductivity on the composition of mixtures of poly(PA)-W with organic acceptors. DDQ, TCNQ, and chloranil were used as organic acceptors. The electrical conductivities for mixtures of poly(PA)-W with DDQ or with TCNQ increased with an increase in the amount of acceptors. This increase is especially remarkable when the acceptor content is $25 \mathrm{~mol} \%$, and a maximum of $c a \cdot 10^{-8} \mathrm{ohm}^{-1} \mathrm{~cm}^{-1}$ is observed around an acceptor content of $75 \mathrm{~mol} \%$. Consequently, a suitable amount of DDQ or TCNQ enhanced the electrical conductivity of poly(PA)-W by $10^{6}$ times. The reason why the electrical conductivity was reduced by the addition of small amounts of DDQ is not clear at present. No incease in conductivity was observed with chloranil, a weaker acceptor.

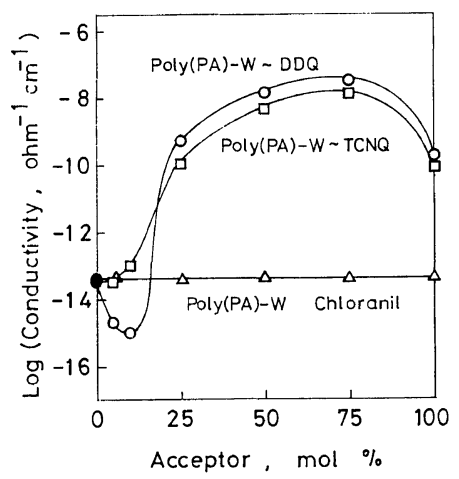

Figure 3. Relationship between the composition and conductivity of mixtures of poly(PA)-W with organic acceptors.

Poly(PA)-Mo is known to possess more cis structure than poly(PA)-W does. ${ }^{14}$ Figure 4 shows that the conductivity of poly(PA)-Mo is not more than $10^{-17} \mathrm{ohm}^{-1} \mathrm{~cm}^{-1}$, and that the conductivity increases to about $10^{-8} \mathrm{ohm}^{-1} \mathrm{~cm}^{-1}$, as the amount of DDQ or TCNQ is increased. Thus, the addition of the acceptors to poly(PA)-Mo resulted in an increase in conductivity by a factor of $10^{9}$. The most considerable increase was observed between 0 and 


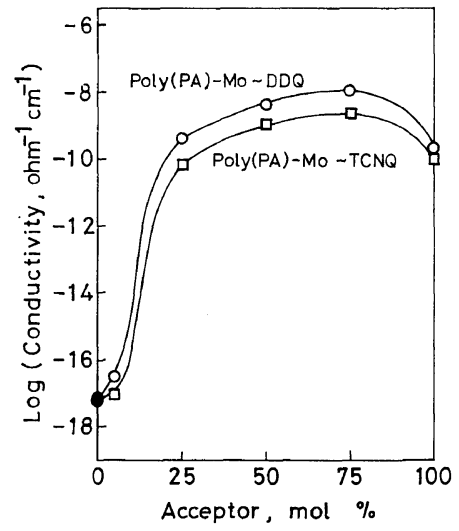

Figure 4. Relationship between the composition and conductivity of mixtures of poly(PA)-Mo with organic acceptors.

\section{$25 \mathrm{~mol} \%$ of acceptor content.}

The effect of acceptors was examined with homologs of poly(PA). Poly( $\beta$-NA), which is also a polymer of monosubstituted acetylene, behaved in a manner similar to poly(PA) (see Figure 5). On the other hand, no maximum was observed in the composition-conductivity relationship of poly(PP). This seems to be concerned with the low conjugation of double bonds in poly(PP) due to the presence of two substituents on a monomer unit. This low conjugation also explains the fact that $\operatorname{poly}(\mathrm{PP})$ is white whereas poly(PA) and $\operatorname{poly}(\beta-\mathrm{NA})$ are yellow to dark brown. ${ }^{16}$

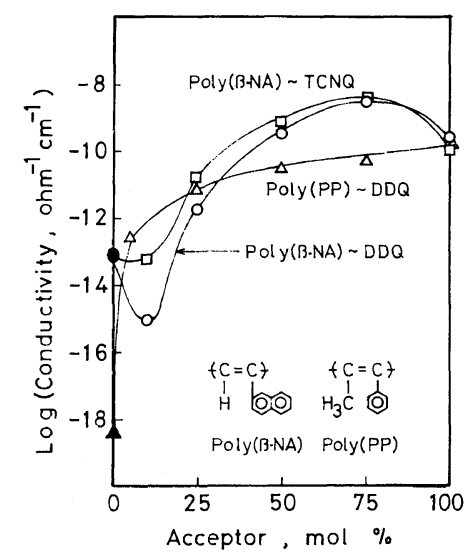

Figure 5. Relationship between the composition and conductivity of mixtures of poly(PA) homologs with organic acceptors.
In an attempt to make clear the effect of conjugated polyene structure, polystyrene was used as a donor polymer. As is clear from Figure 6, the electrical conductivity increased with an increase in the amount of DDQ or TCNQ, but no maximum was observed. While the highest electrical conductivity of the poly(PA)-W $\sim \mathrm{DDQ}$ mixture exceeded $10^{-8} \mathrm{ohm}^{-1} \mathrm{~cm}^{-1}$, that of the polystyreneDDQ mixture was $10^{-10} \mathrm{ohm}^{-1} \mathrm{~cm}^{-1}$. However, this increase in conductivity is also appreciably large, suggesting that an interaction exists between the phenyl ring of polystyrene and the acceptors. It is noted that poly $(\mathrm{PP})$ behaves very similarly to polystyrene with respect to the compositionconductivity relationship.

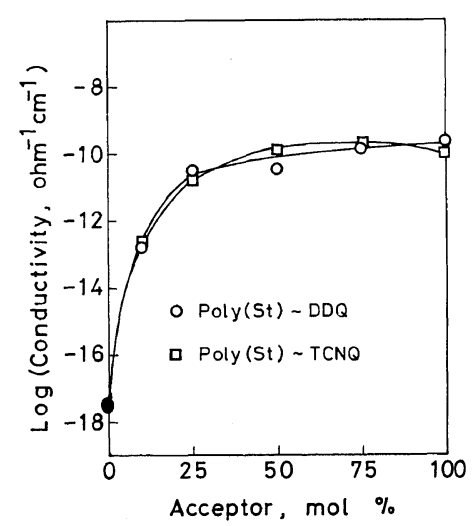

Figure 6. Relationship between the composition and conductivity of mixtures of polystyrene with organic acceptors.

Electrical conductivity for mixtures of poly(PA)$\mathrm{W}$ or poly(PA)-Mo with bromine was investigated. As mentioned above, the bromine content in the mixtures could not be more than $20 \mathrm{~mol} \%$. The conductivities of mixtures whose bromine content was $5-20 \mathrm{~mol} \%$ were as low as $10^{-17}$ to $10^{-15}$ $\mathrm{ohm}^{-1} \mathrm{~cm}^{-1}$, and lower than their original polymers. A mixture of poly(PA)-W with trifluoromethanesulfonic acid $\left(10 \mathrm{~mol}^{\circ} \%\right)$ also exhibited a conductivity as low as $10^{-17} \mathrm{ohm}^{-1} \mathrm{~cm}^{-1}$. These results contrast with those obtained with organicacceptors like DDQ and TCNQ.

It is concluded that current flows predominantly through the species formed from the electron acceptors in the poly(PA) complexes, according to the following reasons: (i) The thermoelectric-power 
measurement on the poly(PA)-W DDQ $(1: 1)$ mixture gave a negative Seebeck coefficient, that is, an $n$-type conduction was confirmed; (ii) Unlike the complexes with bromine and trifluoromethanesulfonic acid, the complexes with organic acceptors were more conducting than poly(PA) itself, probably because of the formation of "complex anions" such as $(\mathrm{DDQ})_{2}{ }^{-}$and $(\mathrm{TCNQ})_{2}{ }^{-}$which function as electron carriers; (iii) Addition of a small amount of acceptors did not lead to a large increase in conductivity, which might deny the $p$-type character of conductivity of the complexes.

As described in the Introduction, polyacetylene doped with small amounts of inorganic electron acceptors is highly conducting. ${ }^{6-9}$ It is noteworthy that the poly(PA) complexes in the present study showed an $n$-type character, while the polyacetylene complexes are $p$-type conductors. ${ }^{8}$ The reason why the poly(PA) complexes cannot be the $p$-type seems to be that the bulky phenyl groups hamper the conjugation of the double bonds along main chain.

In conclusion, the soluble poly(PA)s obtained with $\mathrm{WCl}_{6}$ and $\mathrm{MoCl}_{5}$ formed complexes with organic and inorganic acceptors, and the complexes with organic acceptors showed semiconductivity.

Acknowledgment. The authors wish to thank Dr. Y. Shirota, Osaka University, for his helpful comments. Thanks are also due to Mr. T. Ohtori for rendering technical assistance. This work was supported in part by a Grant-in-Aid for Scientific Research from the Ministry of Education, Science and Culture (No. 355393).

\section{REFERENCES}

1. J. E. Katon, "Organic Semiconducting Polymers," Marcel Dekker Inc., New York, N.Y., 1968.
2. E. P. Goodings, Chem. Soc. Rev., 95 (1976).

3. W. Slough, Trans. Faraday Soc., 58, 2360 (1962).

4. S. B. Mainthia, P. L. Kronick, and M. M. Labes, $J$. Chem. Phys., 41, 2206 (1964).

5. A. M. Hermann, A. Rembaum, J. Polym. Sci., C, 17, 107 (1967).

6. H. Shirakawa, E. J. Louis, A. G. MacDiarmid, C. K. Chiang, and A. J. Heeger, J. Chem. Soc., Chem. Commun., 578 (1977).

7. C. K. Chiang, M. A. Druy, S. C. Gau, A. J. Heeger, E. J. Louis, A. G. MacDiarmid, Y. W. Park, and H. Shirakawa, J. Am. Chem. Soc., 100, 1013 (1978).

8. C. K. Chiang, S. C. Gau, C. R. Fincher, Jr., Y. W. Park, A. G. MacDiarmid, and A. J. Heeger, Appl. Phys. Lett., 33, 18 (1978).

9. Y. W. Park, M. A. Druy, C. K. Chiang, A. G. MacDiarmid, A. J. Heeger, H. Shirakawa, and S. Ikeda, J. Polym. Sci., Polym. Lett. Ed., 17, 195 (1979).

10. T. Masuda, K. Hasegawa, and T. Higashimura, Macromolecules, 7, 728 (1974), and subsequent papers.

11. T. Masuda, H. Kawai, T. Ohtori, and T. Higashimura, Polym. J., 11, 813 (1979), and references therein.

12. H. Noguchi and S. Kambara, J. Polym. Sci., Polym. Lett. Ed., 1, 553 (1963).

13. R. J. Kern, J. Polym. Sci., A-1, 7, 621 (1969).

14. T. Masuda, N. Sasaki, and T. Higashimura, Macromolecules, 8, 717 (1975).

15. T. Ohtori, T. Masuda, and T. Higashimura, Polym. J., 11, 805 (1979).

16. N. Sasaki, T. Masuda, and T. Higashimura, Macromolecules, 9, 664 (1976).

17. R. F. Tarvin, S. Aoki, and J. K. Stille, Macromolecules, 5, 663 (1972).

18. D. S. Acker and W. R. Hertler, J. Am. Chem. Soc., 84, 3370 (1962).

19. T. Natsume, Y. Akana, K. Tanabe, M. Fujimatsu, M. Shimizu, Y. Shirota, S. Kusabayashi, and H. Mikawa, J. Chem. Soc., Chem. Commun., 189 (1969).

20. P. S. Woon and M. F. Farona, J. Polym. Sci., Polym. Lett. Ed., 13, 567 (1975). 